\title{
COMT inhibition with tolcapone in the treatment algorithm of patients with Parkinson's disease (PD): relevance for motor and non-motor features
}

\author{
Angelo Antonini' \\ Giovanni Abbruzzese ${ }^{2}$ \\ Paolo Barone ${ }^{3}$ \\ Ubaldo Bonuccelli ${ }^{4}$ \\ Leonardo Lopiano ${ }^{5}$ \\ Marco Onofrj ${ }^{6}$ \\ Mario Zappia ${ }^{7}$ \\ Aldo Quattrone ${ }^{8}$ \\ 'Parkinson Institute, Istituti \\ Clinici di Perfezionamento, Milan, \\ Italy; ${ }^{2}$ Movement Disorder Unit, \\ Neurology, University of Genoa, \\ Genoa, Italy; ${ }^{3}$ Dipartimento di \\ Scienze Neurologiche, Università \\ di Napoli Federico II, Naples, Italy; \\ ${ }^{4}$ University of Pisa, Department of \\ Neuroscience, Pisa, Italy; ${ }^{5}$ Department \\ of Neuroscience, University of Turin, \\ Turin, Italy; ${ }^{6}$ Department of Oncology \\ and Neuroscience, University \\ G D'Annunzio, G D'Annunzio \\ Foundation CESI, Chieti-Pescara, \\ Italy; ${ }^{7}$ Department of Neurosciences, \\ University of Catania; ${ }^{8}$ Institute \\ of Neurology, University "Magna \\ Græcia," Catanzaro, Italy
}

Correspondence:Angelo Antonini Parkinson Institute, Istituti Clinici di Perfezionamento, 20126 Milano, Italy Tel +390257993222

Fax +390257993319

Email angelo3000@yahoo.com

\begin{abstract}
Levodopa is the most effective treatment in Parkinson's disease and the association with COMT inhibitors widens its plasma bioavailability and effectiveness. Tolcapone is a potent COMT inhibitor whose utilization in PD is limited due to safety concerns on liver toxicity. However, recent data indicate that if liver function is actively monitored, tolerability is no worse than other currently available therapies. By contrast, administration of tolcapone is associated with significant clinical improvement and benefit involves also non-motor features. In this review we discuss the rationale for the use of tolcapone in association with levodopa and other treatments in PD, and we provide an indirect comparison of current strategies to reduce “off" time. We propose that future guidelines include a trial with tolcapone in all PD patients who continue to complain about motor fluctuations despite treatment with entacapone and/or MAO-B inhibitors. Moreover, we suggest that tolcapone should be considered before surgical or infusional strategies are applied.
\end{abstract}

Keywords: Parkinson's disease, levodopa, motor fluctuations, COMT inhibitors, tolcapone

\section{Introduction}

Management of motor fluctuations and dyskinesias is still a major challenge in the long-term treatment of patients with Parkinson's disease (PD) as they affect daily life activities and in turn quality of life of patients and caregivers. Symptoms associated with the "off phase" not only include akinesia, rigidity, and tremor, but also depression, anxiety or even panic attacks (Lauterbach 2005). In the advanced stages of the disease both motor and non-motor fluctuations are present (Nutt and Holford 1996).

Dyskinesias are classified according to their temporal profile after drug administration, namely peak-dose dyskinesias (mainly choreic movements), biphasic dyskinesias, onset and end-of-dose (mainly dystonic and ballic movements), and finally “off”-period dyskinesias (dystonic movements) (Defebvre 2004).

In general motor fluctuations and dyskinesia develop in about $10 \%$ of patients per year after starting levodopa treatment, reaching almost $100 \%$ of patients after 10 years' treatment (Marsden and Parkes 1997; Van Laar 2003). The DATATOP study reported high rates of motor fluctuations and dyskinesias (PSG 1996), but this might be related to direct patient observation during the trial phase (Lang and Lozano 1998).

Recently, a retrospective analysis of the CALM-PD study reported an incidence of motor fluctuations within a range from $12 \%$ to $60 \%$, and an incidence of dyskinesias from $8 \%$ to $64 \%$, after 4-6 years of levodopa therapy (Hauser et al 2006).

Factors involved in the development of motor complications are not completely elucidated yet. Patients with motor fluctuations (wearing off, on-off phenomenon) may show akinetic-rigid onset, longer disease and treatment duration, higher intake of levodopa dose per day (>300 mg/day), and occasionally longer time latency since 
diagnosis. They also show greater disability according to the Schwab-England scale and disease severity. The finding of a family history of parkinsonism is also frequent, suggests that positive genetic may lead to greater propensity to development of motor complications. Similarly patients with dyskinesias tend to present higher Hoehn and Yahr score and longer treatment duration, while association with levodopa daily dose is less consistent. Gender as well as genetic factors related to dopamine D2 receptor gene may also play an important role and contribute to development of peak-dose dyskinesia in women (Schrag and Quinn 2000; Zappia et al 2005).

Although fluctuations in response to levodopa are typically defined by changes in motor signs, autonomic and psychological fluctuations may also occur.

Non-motor fluctuations (NMF) are extremely disabling for PD patients; they affect activity of daily living and quality of life and have recently received growing attention by movement disorders experts. Similar to motor fluctuation, non-motor fluctuations also tend to increase with disease progression, and are more disabling in younger patients (Riley and Lang 1993; Witjas et al 2000).

Non-motor fluctuations may involve cognitive, psychiatric, sensory/pain, and autonomic domains (Chaudhuri et al 2006, 2007).

Assessment of frequency and disability caused by NMF was evaluated in $50 \mathrm{PD}$ patients by the administration of a specific questionnaire (Maricle et al 1998). All patients showed at least one type of NMF, mostly associated with the "off" state. The most represented were anxiety (66\%), drenching sweats (64\%), slowness of thinking (58\%), fatigue (56\%), and akathisia (54\%). All NMF contributed to the worsening motor disability, and especially fluctuations linked to autonomic functions were also related to levodopa treatment.

Some degree of sadness and mood switching is also frequently reported during "on-off” periods (Martinez-Martin et al 2007). Mood fluctuations may occur more frequently in patients with involvement of cortical or mesolimbic areas (Chaudhuri et al 2006).

The stringent relationship between levodopa response and non-motor fluctuations is also supported by the occurrence of "off period" depressive symptoms after discontinuation of levodopa intravenous infusion (Maricle et al 1995, 1998).

Finally, a PET study in a small number of PD patients showed that $\mathrm{rCBF}$ response to levodopa in medial frontal gyrus and posterior cingulated cortex (PCC), was different between mood fluctuators and controls (Black et al 2005). As
PCC is strongly linked to emotions and mood, and it is known to modulate dopaminergic innervations of caudate nucleus, anterior cingulated cortex, and orbital frontex, the authors speculate that mood fluctuations in PD might be related to dysfunction in levodopa responsiveness.

\section{Levodopa administration and response to levodopa: addressing indication to starting therapy}

Levodopa is the most effective treatment of PD cardinal symptoms (rigidity, akinesia, tremor, postural instability) (Rascol et al 2002). The onset of effect is rapid, and most clinical trial results suggest that initiating treatment provides superior motor and functional control compared with other dopaminergic substances. Levodopa is generally well tolerated, with lower incidence of gastrointestinal and neuropsychiatric adverse events (Halkias et al 2007).

The mechanism of action is not simply characterized by a central conversion of levodopa to dopamine at surviving dopaminergic terminals, but also by a neuromodulation (Opacka-Juffry and Brooks 1995), and likely internalization of D1 receptors, as demonstrated in rats (Muriel et al 2002). Moreover, increasing motor fluctuations in the afternoon and evening in patients during long-term levodopa therapy may not relate to the pharmacokinetics of the drug but express tolerance to repeated doses of levodopa (Bonuccelli et al 2000).

Administration of levodopa is associated with negative perception on motor complications risk, leading to a delay in initiating treatment that has been defined as "levodopa phobia".

The relevance of patient age at disease onset for starting levodopa therapy is still debated and current guidelines have not yet properly assessed this issue. The problem of starting levodopa treatment early arises in younger patients with mild motor dysfunction, in consideration of life expectancy and disease progression. Because of its greater efficacy than dopamine agonists, levodopa is recommended as first-line agent always in case of a rapid and sustained worsening of motor symptoms, regardless of age (Olanow et al 2001).

The declining efficacy of levodopa therapy surely recognizes, at least in part, a progression of the disease, and this must be considered especially in terms of expectations on quality of life.

Some hypotheses around the mechanisms by which response fluctuations may occur with levodopa have been conceived (Van Laar 2003): 
1) presynaptic neuronal degeneration leading to insufficient buffering of released levodopa, which could be mainly related to the "wearing off" phenomenon.

2) postsynaptic changes in dopamine receptor sensitivity and number, partially caused by presynaptic changes, which could be related to the unpredictable fluctuations.

3) pharmacokinetic and pharmacodynamic influence of other dopaminergic agents or non-pharmacological agents (such as food).

\section{Clinical response to levodopa can identify at least three different mechanisms}

The best known is the short-duration response (SDR), measured in minutes to hours, with a steep concentration response relationship such that the response appears "all or nothing". The duration of the effect is dose-responsive. During chronic therapy, the SDR becomes shorter, as does the onset to effect, whereas the magnitude of the response becomes larger, probably because of a sensitization phenomenon (Nutt 1995). With regard to tolerance, experiments on levodopa holidays of 2-4 days, demonstrated that post-holiday infusion produced a longer response than did pre-holiday infusion, as measured by tapping and walking speed, tremor, and dyskinesias. The prolongation of the response was greater in patients receiving higher daily doses of levodopa (Nutt et al 1994).

The long-duration response (LDR) results from repetitive administrations of levodopa and develops over days or weeks after starting treatment (Zappia et al 1999a). It is independent of single intakes of levodopa which, in turn, determine SDRs associated with motor fluctuations and dyskinesia (Zappia et al 1997). On the contrary, the presence of the LDR may ensure a stable clinical benefit and its disappearance may be relevant for the development of a fluctuating condition (Zappia et al 1999b). The LDR is more evident in early PD stages, but it could be induced also in more advanced stages (Quattrone et al 1995), by modulating the inter-dose intervals and the size of each dose (Zappia et al 2000). The rate of decay is proportional to the severity of illness, and therefore it might be related to the dopamine storage capacity of surviving nerve terminals. Finally in advanced stages of the disease, we can also identify a negative response (super-off), measured in minutes to hours, and consisting of motor function worsening below the basal level.

\section{Strategies to widen levodopa therapeutic window}

The management of motor and non-motor fluctuations aims at prolonging the effect of dopaminergic stimulation possibly reducing total levodopa load. The effects of a single dose of levodopa may shorten to less than 2-3 hours in advanced patients. Early symptoms of "wearing off" may be improved by redistributing levodopa dosing during the day and shortening the interval between doses (Widnell and Cornella 2005). Alternatively, as modifications of LDR may underline motor fluctuations (Zappia et al 1999a, b), strategies that involve increasing single levodopa dose rather than number of administrations might also be beneficial, if dyskinesia does not worsen (Zappia et al 2000).

There are different strategies aimed at stimulating dopamine receptors in a more continuous, less pulsatile manner, to slow levodopa metabolism, interacting at different levels, and consequently providing a more sustained central effect.

Continuous intraduodenal infusion produces stable levodopa plasma levels resulting in few off periods and dyskinesia as well as improved quality of life, but it can be applied only in limited cases (Antonini et al 2007).

Slow-release preparations of levodopa (Sinemet $\mathrm{CR}^{\circledR}$, Madopar $\mathrm{HBS}^{\circledR}$ ), associated with peripheral dopa decarboxylase inhibitor (DDCI), may be helpful in smoothing out fluctuations, alleviating tremor and rigidity during the night, and morning akinesia as well. However, their absorption and effect is often erratic making their use in a clinical setting very limited.

\section{The MAO-B inhibitors: effectiveness and rationale for use in clinical setting}

MAO-B inhibitors (selegiline, rasagiline), may be an alternative to levodopa in the early treatment (Goetz et al 2005). Although no medication has been proven to slow the progression of the disease, preclinical studies have demonstrated some neuroprotective effects of MAO-B blockers in animal experimental models. These drugs are generally well tolerated, although some concerns have been expressed with selegiline regarding cardiovascular effects (Lees 2005).

Three recent level 1 studies have provided new data on MAO-B rationale for clinical use. In 473 untreated early PD patients allocated to treatment with dopamine agonists (bromocriptine and lisuride), or to selegiline (Caraceni and Musicco 2001), the authors found that the lower frequency of motor complications in patients assigned to selegiline was not statistically significant after a multivariate analysis including effects of age, disease duration, and baseline severity scores.

Shoulson et al (2002) performed an independent randomization of 368 patients from the DATATOP original 
cohort, who had been treated with selegiline and thereafter required levodopa, continuing with selegiline or switching to placebo under double-blind conditions. Patients were followed for 2 years, and primary outcome was the time to the first development of "wearing-off" "on-off" fluctuations, dyskinesia and freezing of gait; confusion and dementia were included in the analysis as well. The frequency of new occurrence in any primary variables was not significantly different between the two arms.

Rasagiline, a second-generation, selective, reversible MAO-B inhibitor, is associated with benefit in patients with early disease stage and reduced "off" time in patients with motor fluctuations (Oldfield et al 2007). According to the TEMPO study (PSG 2002), rasagiline is beneficial as monotherapy in the early stage of the disease. Similar benefit was reported in the LARGO study that compared the effect of entacapone and rasagiline in advanced PD (Rascol et al 2005). Interestingly, a significant mean reduction in "off" time was reported only in patients with mild to moderate dyskinesia but no advantage compared to placebo was found in the most severe advanced PD cohort. These findings confirm the difficulties that current strategies encounter in the management of this specific group of patients.

\section{COMT inhibition with entacapone and tolcapone}

Association of DDCIs with levodopa increases its metabolism by catechol-O-methyltransferase to 3-O-methyl-dopa (3-OMD), in organs expressing COMT activity, such as liver, kidney, and gastrointestinal tract.

The 3-OMD metabolite has a long elimination half-life (approximately 15 hours), and may accumulate during long-term levodopa therapy. 3-OMD may compete with levodopa for passage across the BBB; moreover cerebral COMT metabolize levodopa to 3-OMD and dopamine to homovanillic acid (Kaakkola 2000). On the basis of this knowledge, the role of COMT inhibition seems crucial in PD therapy. Peripheral COMT inhibition reduces degradation of levodopa to 3-OMD, thus widening its therapeutic window, while a central COMT inhibition may further prolong levodopa effectiveness by reducing both levodopa and dopamine metabolism.

The new generation of COMT inhibitors entacapone and tolcapone are potent and selective and their role has become increasingly evident in the last decade (Figure 1).

The two drugs show a number of pharmacologic differences and consequently, different clinical effectiveness.
Tolcapone is a selective, reversible peripheral COMT inhibitor (Napolitano et al 1999). Activity on central COMT has also been demonstrated both clinically and with functional imaging (Ceravolo et al 2002) and it can be observed for doses of $200 \mathrm{mg}$ tid.

Data about efficacy and safety of these drugs have been examined recently by a Cochrane review (Deane et al 2004). Comparing the efficacy of the two COMT inhibitors across 8 randomized controlled trials, tolcapone produced larger reductions in "off" time and levodopa requirements.

Tolerability profile was similar; some of the reported adverse events were related to the increased availability of levodopa (nausea, vomiting, dyskinesias, hallucination), and ameliorated reducing levodopa dose. Some other adverse events were more strictly related to COMT-inhibition, in particular the occurrence of diarrhea.

\section{Role of tolcapone in PD management}

Tolcapone, a potent selective reversible COMT inhibitor, was launched in Europe in 1997, and in the US in 1998 (Leegwater-Kim and Waters 2006). Its efficacy as adjunct to levodopa therapy is attributed to the peripheral COMT inhibition, whereas there is still some debate around clinical relevance associated to the central activity. Results of clinical trials conducted in PD patients, confirm that tid administration of tolcapone magnifies levodopa motor benefit. Improvement had already been observed with a single $200 \mathrm{mg}$ dose (Napolitano et al 1999).

Compared with levodopa/DDCI alone, single or multiple doses of tolcapone 100 or $200 \mathrm{mg}$ increased levodopa bioavailability by 1.3 to 2.1 fold, both in healthy volunteers and in PD patients (Limousin et al 1995).

Pharmacological profile of tolcapone is characterized by a greater bioavailability, and so has a greater area under the curve, $\mathrm{T}_{\text {max }}$ and COMT enzyme affinity, compared with entacapone. Tolcapone increases the levodopa half-life by $80 \%$ versus $40 \%$ for entacapone, the levodopa area under the curve by $80 \%$ versus $40 \%$ for entacapone, providing a greater decrease in the formation of O-methyl-dopa as well (Factor et al 2001).

The results of a long-term comparative study between tolcapone and entacapone, suggest that tolcapone is more effective, and this difference in response would be predicted by the pharmacological differences between the two agents (Onofrj et al 2001). The study, performed in patients with fluctuating PD, compared efficacy and tolerability of tolcapone and entacapone, evaluating the effects prospectively at $6,12,24$, and 36 months. Fourteen patients were enrolled 


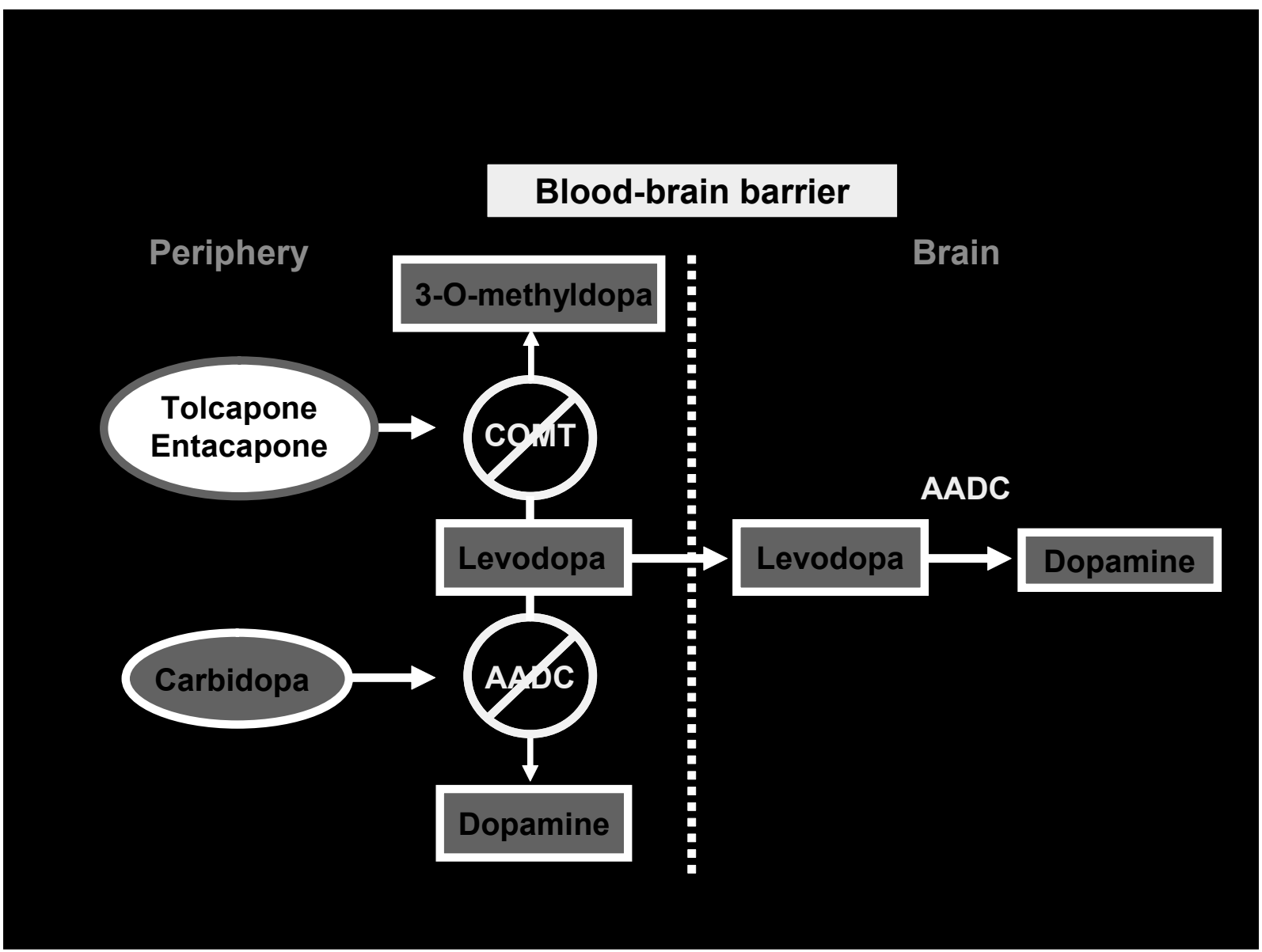

Figure I COMT-I: mechanism of action.

under tolcapone treatment, and eleven patients were enrolled under entacapone treatment. Tolcapone showed more effectiveness in lowering UPDRS motor score and complication subscores, duration of "off" periods, and levodopa doses requirement (UPDRS motor score change was $-5.07 \pm 1.48$ in tolcapone group, and $+2.46 \pm 2.76$ for entacapone $(\mathrm{p}<0.02)$; duration of "off" period was $-1 \pm 0.23$ for tolcapone, $0 \pm 0.27$ for entacapone ( $\mathrm{p}<0.03)$, lowering of L-DOPA dose was $-400 \pm 70.7$ for tolcapone and $+179.2 \pm 160.8$ for entacapone $(\mathrm{p}<0.04)$.

Another study in 40 patients with severe PD, with open design and direct switch-over from tolcapone to entacapone, was prompted because of mandatory indications of the European drugs authority, who withdrew tolcapone from market in November 1998. "On" time was increased by $15 \%$ during tolcapone treatment $(\mathrm{p}<0.05)$, and by $8 \%$ during entacapone treatment, whereas "off" time was decreased by $16 \%$ during tolcapone and by $7 \%$ during entacapone treatment. The reduction of L-DOPA was significantly improved by tolcapone compared with entacapone $(p=0.05)$ (Lees et al 2007). IGA (Investigator Global Assessment) for wearing off severity, indicated that $70-73 \%$ of the patients receiving tolcapone, and $56 \%$ of patients receiving entacapone, had a relevant improvement $(\mathrm{p}=0.01)$.

A recent double blind study assessed patients receiving entacapone after random assignement to continue entacapone $(n=75)$ or switch to tolcapone $(n=75)$. The "on" time increased by $\geq 1$ hour day in $43 \%$ of entacapone patients and in $53 \%$ of tolcapone patients and by $\geq 3$ hours day in $13 \%$ and $25 \%$ of patients, respectively. The IGA indicated moderate/marked improvement in $25 \%$ of entacapone patients and 39\% receiving tolcapone. No differences were observed in the rate of dyskinesias and adverse events leading to discontinuation of treatment (Pahwa et al 2006).

Safety and tolerability were evaluated with a focus on liver transaminase elevations and hepatotoxicity; 677 levodopa-naïve patients were randomized to receive placebo or tolcapone as add-on therapy to standard doses of levodopa 
Table I Efficacy of oral tolcapone 100 mg, entacapone, and rasagiline evaluated in terms of increasing hours of "on" time, decreasing hours of "off" time, and levodopa requirement

\begin{tabular}{|c|c|c|c|c|c|c|c|}
\hline \multirow[t]{2}{*}{ Study } & \multirow{2}{*}{$\begin{array}{l}\text { Study drug dosage } \\
\text { (mg/tid) }\end{array}$} & \multirow{2}{*}{$\begin{array}{l}\text { Study } \\
\text { duration }\end{array}$} & \multirow{2}{*}{$\begin{array}{l}\mathbf{N} \text { of } \\
\text { patients }\end{array}$} & \multicolumn{2}{|c|}{ Mean change from baseline: } & \multicolumn{2}{|l|}{ Daily levodopa dosage: } \\
\hline & & & & “on" time & "off" time & Baseline (mg/day) & \%change from $b$ \\
\hline \multirow[t]{2}{*}{ Baas et al 1997} & Tolcapone 100 & $3 \mathrm{mo}$ & 60 & $+1.7 \mathrm{~h}^{* *}$ & $-2 h^{*}$ & 667.5 & $-16 *$ \\
\hline & Placebo & & 58 & $-0.1 \mathrm{~h}$ & $-0.7 \mathrm{~h}$ & 660.5 & -4 \\
\hline \multirow[t]{2}{*}{ Rajput et al I997 } & Tolcapone 100 & $3 \mathrm{mo}$ & 69 & - & $-2.3 \mathrm{~h}$ & 788.5 & $-2 I^{* *}$ \\
\hline & Placebo & & 66 & & $-\mathrm{I} .4 \mathrm{~h}$ & 948.0 & -2 \\
\hline \multirow[t]{2}{*}{ Adler et al 1998} & Tolcapone 100 & 6 wks & 69 & $+2.1 \mathrm{~h} * * *$ & $-2.0 \mathrm{~h}^{* * *}$ & 810 & $-23 * *$ \\
\hline & Placebo & & 72 & & & 850 & $>-1$ \\
\hline \multirow[t]{2}{*}{ Shan et al 200I } & Tolcapone 100 & 6 wks & 20 & - & $-15.8 \% *$ & 795.0 & -7 \\
\hline & Placebo & & 20 & & $+1.2 \%$ & 930.0 & -2 \\
\hline \multirow[t]{2}{*}{ SWITCH 2006} & Tolcapone 100 & 3 wks & 150 (tot) & $+1.6 *$ & - & - & - \\
\hline & Entacapone 200 & & & +0.8 & & & \\
\hline \multirow[t]{4}{*}{ Onofrj et al 200I } & Tolcapone 100 & $3-7$ mo & 40 (tot) & $+15 \% *$ & $-16 \%$ & Levodopa was sig- & \\
\hline & Entacapone 200 & & & $+8 \%$ & $-7 \%$ & nificantly reduced with & \\
\hline & & & & & & tolcapone: $\mathrm{p}=0.0 \mathrm{I}$ and & \\
\hline & & & & & & entacapone: $p=0.05$ & \\
\hline \multirow[t]{7}{*}{ Rascol 2005} & Rasagiline (I mg/day) & 18 wks & 231 & $+0.85 \mathrm{~h}$ & $-1.18 \mathrm{~h}$ & & \\
\hline & Entacapone $(200$ mg/ & & 200 & $+0.03 \mathrm{~h}$ & $-\mathrm{I} .2 \mathrm{~h}$ & & \\
\hline & with every levodopa & & 229 & $(p=0.0005$ & $-0.4 \mathrm{~h}$ & & \\
\hline & dose) & & & for both) & $(p=0.0001$ & & \\
\hline & Placebo & & & & $\mathrm{p}<0.000 \mathrm{I}$ & & \\
\hline & & & & & respectively vs & & \\
\hline & & & & & placebo) & & \\
\hline \multirow[t]{3}{*}{ PSG 2005} & Rasagiline $0.5 \mathrm{mg} /$ day & 26 wks & 164 & +0.51 & -0.49 & & \\
\hline & Rasagiline I mg/day & & 149 & $+0.78^{*}$ & $-0.94 * *$ & & \\
\hline & Placebo & & 159 & & & & \\
\hline
\end{tabular}

${ }_{\mathrm{p}}<0.05,{ }^{*} \mathrm{p}<0.01,{ }^{* * *} \mathrm{p}<0.001$ vs placebo, ${ }^{* * * * *} \mathrm{p}=0.018$

(The Entacapone to Tolcapone Switch Study Investigators 2007). Liver transaminase elevations above the upper normal limit (ULN) occurred in $20.2 \%$ and $27.5 \%$ of patients in the placebo and tolcapone groups, respectively. Elevations of $\mathrm{ULN} \geq 3$ fold occurred in $1.2 \%$ and $1.8 \%$ of placebo and tolcapone patients, respectively. No serious hepatotoxicity events were reported.

Efficacy of oral tolcapone as adjunct therapy to levodopa/DDCI, was demonstrated in several trials, although only in the short period (3 weeks' to 3 months' duration), both in fluctuating and stable PD patients (Suchowersky et al 2001).

Clinical trials conducted in PD patients with motor fluctuations (wearing off, predictable “on-off”), were analyzed by a Committee of the American Academy of Neurology. The Committee recognized tolcapone is useful in reducing the "off" time, the need of levodopa daily dose, and prolonging the "on" phase.

Recently, also data from a comparator study (the SWITCH study) were presented. The aim of the study was to determine if switching from entacapone to tolcapone improves motor features. In the per-protocol analysis, a statistically significant greater proportion of patients switched to tolcapone, had at least 3 hours/day of additional "on" time $(\mathrm{p}=0.018)$. More patients on tolcapone were judged to have a moderate or marked improvement in the IGA, although not statistically significant. Also the number of responders was higher on tolcapone, which appeared to have greater long-term benefits compared to entacapone (Table 1).

In patients with stable disease, tolcapone determines improvement from baseline in various end-points in a short-term study (Suchowersky et al 2001), showing an overall global improvement in $77 \%$ of patients at the IGA score, after 4 weeks double-blind treatment.

These preliminary results suggest potential benefit on both motor and non-motor fluctuations. In this respect, patient-perceived benefit, obtained by smoother levodopa response, might be regarded also as an indirect effect of improved non-motor fluctuations. 
With regard to safety, dopaminergic-related adverse events were the most frequently reported in patients receiving $200 \mathrm{mg}$ tolcapone tid. Such events (nausea, vomiting, dyskinesia, orthostatic complaints, sleep disorders, dystonia, confusion, hallucination), are likely a consequence of the increase in levodopa concentrations, especially at the beginning of treatment, and are mostly resolved with a reduction in levodopa dose. New or worsening dyskinesias were one of the most commonly reported dopaminergic-related adverse events (25\%-62\% with tolcapone, $100 \mathrm{mg} 3$ times daily, $31 \%-66 \%$ with $200 \mathrm{mg} 3$ times daily); in any case they were not a reason for withdrawal (Deane et al 2004). Some major concerns have been raised in the past over the emergence of liver toxicity (Watkins 2000).

For this reason, marketing authorization for tolcapone was suspended in the EU (late 1998), and labeling was tightened in the US. However, suspension of the marketing authorization in the EU was later lifted following EMEA recommendation (EMEA 2004).

The cause of hepatotoxicity has not been clearly established yet, although the current hypothesis suggests a possible interference with mithocondrial respiration in hepathocytes, by uncoupling oxidative phosphorilation (Borges 2005).

Three cases of fatal fulminant hepatic failure were reported with tolcapone in 1998, but it should be noted that liver function had not been properly monitored in these patients and that the liver dysfunction emerged 2-6 months after starting treatment (Olanow 2000). A recent review of tolcapone global safety (Valeant Pharmaceuticals International data on file), reported severe hepatocellular injury only in $0.04 \%$ of patients over the last 7 years, whereas the majority of patients experiencing liver toxicity, only showed an elevations in liver transaminases without clinical signs or symptoms.

In a review of clinical data on 1535 patients (Olanow 2000), the expert panel also noted that about half the patients who continued tolcapone therapy experienced a normalization of ALT/AST levels within 1-3 months, whereas patients who discontinued tolcapone experienced the normalization after just 2-3 weeks. These data are crucial in the evaluation of tolcapone's role in PD therapy; in fact, due to its fast onset of action, the identifications of responders can occur early, when the liver damage is probably still in a reversible initial phase, so non-responders may easily withdraw before liver damage is established, whereas responders should be strictly monitored, especially in the first 6 months.

In summary, tolcapone is a useful option for patients with idiopathic, fluctuating PD patients (Colosimo 1999).
Clinical practice suggests that tolcapone is more effective than entacapone and it can be successfully associated to levodopa preparations. In addition it has central activity with potential benefits on mood and cognition although this might be observed only at doses of $200 \mathrm{mg}$ tid. Data on tolcapone effect in patients who have not benefited from entacapone (this would be the target population according to EMEA guidelines) are still lacking. Randomized trials are warranted to demonstrate that adding tolcapone in these individuals is indeed beneficial.

\section{Conclusions}

Current evidence suggests that treatment strategies should target both motor and non-motor fluctuations, as the latter have been often neglected or underestimated in PD. COMT inhibitors are beneficial because they widen the therapeutic window in PD. In addition tolcapone may also have some central activity particularly at high dose. Given this evidence we suggest that tolcapone should be prescribed to all PD patients who continue to complain about motor fluctuations despite current or previous treatment with entacapone and/or MAO-B inhibitors. We believe that a tolcapone trial should be considered before other complex and more invasive procedures are proposed to the patient.

\section{References}

Adler CH, Singer C, O’Brien C, et al. 1998. Randomized, placebo-controlled study of tolcapone in patients with fluctuating Parkinson disease treated with levodopa-carbidopa. Tolcapone Fluctuator Study Group III. Arch Neurol, 55:1089-95.

Antonini A, Isaias IU, Canesi M, et al. 2007. Duodenal levodopa infusion for advanced Parkinson's disease: 12-month treatment outcome. Mov Disord, 22:1145-9.

Baas H, Beiske AG, Ghika J, et al. 1997. Catechol-O-methyltransferase inhibition with tolcapone reduces the "wearing off" phenomenon and levodopa requirements in fluctuating parkinsonian patients. $J$ Neurol Neurosurg Psychiatry, 63:421-8.

Black KJ, Hershey T, Hartlein JM, et al. 2005. Levodopa challenge neuroimaging of levodopa-related mood fluctuation in Parkinson's disease. Neuropsychopharmacology, 30:590-601.

Bonuccelli U, Del Dotto P, Lucetti C, et al. 2000. Diurnal motor variations to repeated doses of levodopa in Parkinson's disease. Clin Neuropharmacol, 23:28-33.

Borges N. 2005. Tolcapone in Parkinson's disease: liver toxicity and clinical efficacy. Expert Opin Drug Saf, 4:69-73

Caraceni T, Musicco M. 2001. Levodopa or dopamine agonists, or deprenyl as initial treatment for Parkinson's disease. A randomized multicenter study. Parkinsonism Relat Disord, 7:107-14.

Ceravolo R, Piccini P, Bailey DL, et al. 2002. 18F-dopa PET evidence that tolcapone acts as a central COMT inhibitor in Parkinson's disease. These findings are compatible with clinical doses of tolcapone having a significant blocking effect on peripheral and central COMT but not DDC activity in PD. Synapse, 43:201-7.

Chaudhuri KR, Martinez-Martin P, Brown RG, et al. 2007. The metric properties of a novel non-motor symptoms scale for Parkinson's disease: results from an international pilot study. Mov Disord, 22:1901-11. 
Chaudhuri KR, Martinez-Martin P, Schapira AH et al. 2006; International multicenter pilot study of the first comprehensive self-completed nonmotor symptoms questionnaire for Parkinson's disease: the NMS Quest study. Mov Disord, 21:916-23.

Colosimo C. 1999. The rise and fall of tolcapone. J Neurol, 246:880-2.

Deane KH, Spieker S, Clarke CE. 2004. Catechol-O-methyltransferase inhibitors versus active comparators for levodopa-induced complications in Parkinson's disease. Cochrane Database Syst Rev, 18:2005.

Defebvre L 2004. Motor complications in DOPA treatment of Parkinson's disease: clinical description and evaluation. Therapie, 59:93-6.

European Medicines Evaluation Agency. EMEA public statement on the lifting of the suspension of the marketing authorisation for Tolcapone (Tasmar) (online). Accessed 2004. URL:http//www.emea.eu.int.

Factor SA, Molho ES, Feustel PJ, et al. 2001. Long-term comparative experience with tolcapone and entacapone in advanced Parkinson's disease. Clinic Neuropharmacol, 24:295-9.

Goetz CG, Poewe W, Rascol O, et al. 2005. Evidence based medical review update: pharmacological and surgical treatments of Parkinson's disease: 2001 to 2004. Mov Disord, 20:523-39.

Halkias IA, Haq I, Huang Z, et al. 2007 When should levodopa therapy be initiated in Patients with Parkinson's disease? Drugs Aging, 24:61-73.

Hauser RA, McDermott MP, Messing S. 2006. Factors associated with the development of motor fluctuations and dyskinesias in Parkinson Disease. Arch Neurol, 63:1756-60.

Kaakkola S. 2000. Clinical pharmacology, therapeutic use and potential of COMT inhibitors in Parkinson's Disease. Drugs, 59:1233-50.

Lang AE, Lozano AM. 1998. Parkinson's disease. Second of two parts (Review). N Engl J Med, 339:1130-43.

Lauterbach EC. 2005. The neuropsychiatry of Parkinson's disease. Minn Med, 96:155-73.

Leegwater-Kim J, Waters C. 2006. Tolcapone in the management of Parkinson's disease. Expert Opin Pharmacother, 7:2263-70.

Lees AJ. 2005. Alternatives to levodopa in the initial treatment of early Parkinson's disease. Drugs Aging, 22:731-40.

Lees AJ, Ratziu V, Tolosa E, et al. 2007. Safety and tolerability of adjunctive tolcapone therapy in early Parkinson's disease patients. J Neurol Neurosurg Psychiatry, 78:944-8.

Limousin P, Pollak P, Pfefen JP, et al. 1995. Acute administration of levodopa-beserazide and Tolcapone, a COMT inhibitor, in Parkinson's disease. Clin Neuropharmacol, 18:258-65.

Maricle RA, Nutt JG, Carter JH. 1995. Mood and anxiety fluctuation in Parkinson's disease associated with levodopa infusion: preliminary findings. Mov Disord, 10:329-32.

Maricle RA, Valentine RJ Carter J, et al. 1998. Mood response to levodopa infusion in early Parkinson's Disease. Neurology, 50:1890-2.

Martinez-Martin P, Chaudhuri KR, Stocchi F et al. 2007. Prevalence of non-motor symptoms in Parkinson's disease in an international setting; Study using non-motor symptoms questionnaire in 545 patients. Mov Disord, 22:1623-9.

Muriel MP, Orieux G, Hirsch EC. 2002. Levodopa but not ropinirole induces an internalization of D1 dopamine receptors in parkinsonian rats. Mov Disord, 17:1174-79.

Napolitano A, Del Dotto P, Petrozzi L, et al. 1999. Pharmacokinetics and pharmacodynamics of L-Dopa after acute and 6-week tolcapone administration in patients with Parkinson's disease. Clin Neuropharmacol, 22:24-9.

Nutt JG. 1995. Pharmacodynamics of levodopa in Parkinson's disease. Clin Exp Pharmacol Physiol, 22:837-40.

Nutt JG, Carter JH, Woodward WR. 1994. Effect of brief levodopa holidays on the short-duration response to levodopa: evidence for tolerance to the antiparkinsonian effects. Neurology, 44:1617-22.

Nutt JG, Holford NHG. 1996. The response to levodopa in Parkinsons' disease: imposing pharmacological law and order. Annals Neurol, 39:561-73.

Olanow CW. 2000. Tolcapone and hepatotoxic effects. Tasmar Advisory Panel. Arch Neurol, 57:263-7.
Olanow CW, Watts RL, Koller WC. 2001. An algorithm (decision tree) for the management of PD; 2001: treatment guidelines. Neurology, 56(Suppl 5):S1-88

Oldfield V, Keating GM, Perry CM. 2007. Rasagiline: a second generation monoamine oxidase type B inhibitor for the treatment of Parkinson's disease. Drugs, 67:1725-47.

Onofrj M, Thomas A, Iacono D, et al. 2001. Switch-Over from tolcapone to entacapone in severe Parkinson's disease patients. Eur Neurol, 46:11-16.

Opacka-Juffry J, Brooks DJ. 1995. L-dihydroxyphenylalanine and its decarboxylase: new ideas on their neuroregulatory roles. Mov Disord, 10:241-9.

Pahwa R, Factor SA, Lyons KE et al. 2006. Practice parameter: treatment of Parkinson Disease with motor fluctuations and dyskinesia (an evidencebased review): Report of the quality Standards Subcommittee of the American Academy of Neurology. Neurology, 66:983-95.

[PSG] Parkinson Study Group. 1996. Impact of deprenyl and tocopherol treatment on Parkinson's Disease in DATATOP patients requiring levodopa. Ann Neurol, 39:37-45.

[PSG] Parkinson Study Group. 2002. A controlled trial of rasagiline in early Parkinson disease: the TEMPO Study. Arch Neurol, 59:1937-43.

[PSG] Parkinson Study Group. 2005. A randomized placebo-controlled trial of rasagiline in levodopa - treated patients with Parkinson disease and motor fluctuations: the PRESTO study.Arch Neurol, 62:241-8.

Quattrone A, Zappia M, Aguglia U, et al. 1995. The subacute levodopa test for evaluating long-duration response in Parkinson's disease. Ann Neurol, 38:389-95.

Rajput AH, Martin W, Saint-Hilaire MH, et al. 1997. Tolcapone improves motor function in parkinsonian patients with the "wearing-off" phenomenon: a double-blind, placebo-controlled, multicenter trial. Neurology, 49:1066-71.

Rascol O, Goetz C, Koller W, et al. 2002. Treatment interventions for Parkinson's disease: an evidence based assessment. Lancet, 359:1589-98.

Rascol O, Melamed E, Oertel W, et al. 2005. Rasagiline, as an adjunct to levodopa in patients with Parkinson's disease and motor flucuations (LARGO, lasting effect in adjunct therapy with Rasagiline given once daily; study): a randomized, double-blind, parallel-group trial. Lancet, 365:947-54.

Riley DE, Lang AE, 1993. The spectrum of levodopa-related fluctuations in Parkinson's disease. Neurology, 43:1459-64.

Schrag A, Quinn N. 2000. Dyskinesias and motor fluctuations in Parkinson's disease A community-based study. Brain, 123:2297-305.

Shan DE, Lee SJ, Chao LY, et al. 2001. Gait analysis in advanced Parkinson's disease - effect of levodopa and tolcapone. Can J Neurol Sci, 28:70-5.

Shoulson I, Oakes D, Fahn S, et al. 2002. Impact of sustained deprenyl (selegiline) in levodopa-treated Parkinson's disease: a randomized placebo-controlled extension of the deprenyl and tocopherol antioxidative therapy of parkinsonism trial. Ann Neurol, 51:604-12.

Suchowersky O, Bailey P, Pourcher E, et al. 2001. Comparison of two dosages of Tolcapone added to levodopa in nonfluctuating patients with PD. Clin Neuropharmacol, 24:214-20.

The Entacapone to Tolcapone Switch Study Investigators. 2007. Entacapone to tolcapone switch: multicenter double blind, randomized, active controlled trial in Advanced Parkinson's disease. Mov Disord, $22: 14-19$.

Van Laar T. 2003. Levodopa-induced response fluctuations in patients with Parkinson's disease: strategies for management. CNS Drugs, 17:475-89.

Watkins P. 2000. COMT inhibitors and liver toxicity. Neurology, 55(11 Suppl):S51-2.

Widnell KL, Comella C. 2005. Role of COMT Inhibitors and dopamine agonists in the treatment of motor fluctuations. Mov Disord, 20(Suppl 11):30-7.

Witjas T, Kaphan E, Azulay JP, et al. 2000. Nonmotor fluctuations in Parkinson's disease: frequent and disabling. Neurology, 59:408-13. 
Zappia M, Annesi G, Nicoletti G, et al. 2005. Sex differences in clinical and genetic determinants of levodopa peak-dose dyskinesias in Parkinson disease: an exploratory study. Arch Neurol, 62:601-5.

Zappia M, Bosco D, Plastino M, et al. 1999a. Pharmacodynamics of the long-duration response to levodopa in PD. Neurology, 53:557-60.

Zappia M, Colao R, Montesanti R, et al. 1997. Long-duration to levodopa influences the pharmacodynamics of short-duration response in Parkinson's disease. Ann Neurol, 42:245-8.

Zappia M, Olivieri RL, Bosco D, et al. 2000. The long-duration response to L-dopa in the treatment of early PD. Neurology, 54:247-50.

Zappia M, Olivieri RL, Montesanti R, et al. 1999b. Loss of long-duration response to levodopa over time in PD. Implications for wearing-off. Neurology, 52:763-7. 
